\title{
SMALL-SCALE STRUCTURE AT HIGH REDSHIFT. III. THE CLUMPINESS OF THE INTERGALACTIC MEDIUM ON SUBKILOPARSEC SCALES ${ }^{1}$
}

\author{
Michael Rauch, ${ }^{2}$ Wallace L. W. Sargent, ${ }^{3}$ Thomas A. Barlow, ${ }^{3}$ and Robert F. Carswell ${ }^{4}$ \\ Received 2001 March 13; accepted 2001 July 27
}

\begin{abstract}
Spectra obtained with the Keck HIRES instrument of the Ly $\alpha$ forests in the lines of sight to the A and $\mathrm{C}$ components of the gravitationally lensed quasi-stellar object Q1422+231 were used to investigate the structure of the intergalactic medium at mean redshift $\langle z\rangle \sim 3.3$ on subkiloparsec scales. We measured the cross-correlation amplitude between the two Ly $\alpha$ forests for a mean transverse separation of $120 h_{50}^{-1}$ pc and computed the rms column density and velocity differences between individual absorption systems seen in both lines of sight. The rms differences between the velocity centroids of the Ly $\alpha$ forest lines were found to be less than about $400 \mathrm{~ms}^{-1}$ for unsaturated $\mathrm{H}$ I absorption lines with column densities in the range $12<\log N(\mathrm{H} \mathrm{I})<14.13$. The rate of energy transfer into the low-density intergalactic medium on a typical scale of $100 \mathrm{pc}$ seems to be lower by 3-4 orders of magnitude than the rate measured earlier for strong C IV metal absorption systems. The tight correlation between $\mathrm{H}$ I column density and baryonic density in the intergalactic medium was used to obtain a conservative upper limit on the rms fluctuations of the baryonic density field at $\langle z\rangle=3.26$, namely, $\left[\left\langle(\Delta \log \rho)^{2}\right\rangle\right]^{1 / 2} \leq 3.1 \times 10^{-2}$ on a scale of $110 h_{50}^{-1}$ pc. The fraction of the absorption lines that are different across the lines of sight was used to determine the filling factor of the universe for gas that has suffered recent hydrodynamic disturbances. We thereby derived upper limits on the filling factor of galactic outflows at high redshift. Short-lived, short-range ancient winds are essentially unconstrained by this method, but strong winds blowing for a substantial fraction of a Hubble time (at $z=3.3$ ) appear to fill less than $20 \%$ of the volume of the universe.
\end{abstract}

Subject headings: cosmology: observations - gravitational lensing — intergalactic medium quasars: absorption lines - quasars: individual $(\mathrm{Q} 1422+231)$

\section{INTRODUCTION}

Much astronomical effort has been expended to investigate the structure of the universe on ever larger scales. Surveys of galaxies in emission, studies of intergalactic gas clouds in absorption, and searches for anisotropy in the cosmic microwave background indicate a universe approaching homogeneity on scales larger than a few hundred megaparsecs (e.g., Peebles 1993). Velocity and density variations, smoothed over increasingly larger volumes, decrease steadily, in agreement with current cosmological $n$-body and hydrosimulations.

In this paper, we investigate how the density and velocity fluctuations behave on the smallest scales. Even limiting ourselves to randomly chosen regions (which have densities far short of those encountered in stars and other highly collapsed objects) the answer is complex and must depend strongly on the local mean density. Large density fluctuations on very small (parsec) scales occur in the interstellar medium. The intergalactic medium (IGM), at least as observed at high redshifts, occupies an intermediate range between large-scale homogeneity and small-scale fragmentation. The combination of low density and photoioniza-

\footnotetext{
${ }^{1}$ The observations were made at the W. M. Keck Observatory, which is operated as a scientific partnership between the California Institute of Technology and the University of California. It was made possible by the generous support of the W. M. Keck Foundation.

${ }^{2}$ Carnegie Observatories, 813 Santa Barbara Street, Pasadena, CA 91101.

${ }^{3}$ Astronomy Department, California Institute of Technology, Pasadena, CA 91125.

${ }^{4}$ Institute of Astronomy, Cambridge University, Madingley Road, Cambridge CB3 OHA, UK.
}

tion heating by the metagalactic UV background conspire to prevent catastrophic collapse and cooling. In the absence of discrete sources of momentum, heat, or ionization, any small-scale structure below the Jeans mass should be smoothed out by the thermal pressure of the intergalactic gas.

The nature of small-scale structure in the IGM has received renewed interest with the advent of high-resolution gasdynamic simulations of structure formation. It is possible to measure various cosmological and astrophysical quantities by comparing hydrosimulations of the general baryon distribution with observations of Ly $\alpha$ forest absorption lines (e.g., Cen et al. 1994; Petitjean, Muecket, \& Kates 1995; Zhang, Anninos, \& Norman 1995; Hernquist et al. 1996; Miralda-Escudé et al. 1996; Wadsley \& Bond 1996; Zhang et al. 1997; Croft et al. 1997; Theuns et al. 1998; Bryan et al. 1999; Davé et al. 1999). In spite of the successes of the models in reproducing the observed Ly $\alpha$ forest absorption, there is some concern that the finite mass resolution may limit quantitative conclusions as to the thermodynamics and ionization state of the gas. For example, the optical depth of an absorption line for a given value of the background density is approximately proportional to the clumpiness of the gas. Consequently, a locally denser gas could, to some degree, mimic a universe with a larger baryon density and still satisfy the observational constraints (see Rauch et al. 1997; Weinberg et al. 1997). Thus, estimates of the baryon density in the IGM depend on either the simulations properly resolving those low to intermediate column density structures in the IGM that contain most of the baryons or on the absence of any significant small-scale structure. Since the Jeans mass $M_{\mathrm{J}}$ varies proportional to $\rho^{-1 / 2}\left[\propto(1+z)^{-3 / 2}\right]$, there is always a red- 
shift where the minimum resolved mass exceeds $M_{\mathrm{J}}$, so small-scale structure present at high redshift could pose a problem. There are good reasons for concern that such structure may exist at some level. Velocity gradients of several kilometers per second, or appreciable density differences over distances of a few hundred parsecs in low column density gas, might imply that already at high redshift much of the matter is trapped in unresolved, small potential wells; alternatively, the gas may have been stirred by nongravitational agents such as galactic winds and explosions (e.g., Couchman \& Rees 1986). The widespread metal enrichment in the IGM shows that such galactic outflows must have occurred. There have been several theoretical attempts trying to understand this result (e.g., Ferrara, Pettini, \& Shchekinov 2000; Theuns, Mo, \& Schaye 2001; Cen \& Bryan 2001; Madau, Ferrara, \& Rees 2001; Aguirre et al. 2001), but little is known about the hydrodynamic consequences of these ancient winds for the IGM. Finally, it has been argued that even at high redshift many Ly $\alpha$ forest absorption lines are formed in extended galactic halos (Chen et al. 2000), in which case one should expect to see small-scale hydrodynamic disturbances in the gas caused by stellar feedback.

The only way to test for the possibility of hidden smallscale structure and of a universe grainier than envisaged by the simulations is to measure by how much the universal density and velocity fields fluctuate on a given spatial scale. Differences in the absorption pattern of the Ly $\alpha$ forest in closely spaced lines of sight to gravitationally lensed quasistellar objects (QSOs) can be used to measure the clumpiness of the IGM on subkiloparsec scales, about an order of magnitude smaller than can currently be resolved by cosmological simulations. Such observations have been used to establish lower limits on the sizes of the Ly $\alpha$ forest clouds (e.g., Young et al., 1981; Weymann \& Foltz 1983; Foltz et al. 1984; Smette et al. 1993, 1995; Bechtold \& Yee 1995; Petry, Impey, \& Foltz 1998). Most of the above investigations have shown the difference between the lines of sight to be small. In the previous paper (Rauch, Sargent, \& Barlow 2001, hereafter Paper II), the second in a series of papers on the small-scale properties of various gaseous environments at high redshift, we found that the metalenriched gas observed in C IV absorption systems, apparently produced in extended regions around redshift galaxies, shows signs of having been disturbed hydrodynamically on timescales similar to those relevant for recurrent star formation. This is consistent with the C IV gas clouds being the result of ancient galactic outflows. The C IV absorbing gas is mostly related to strong saturated Ly $\alpha$ forest systems, and the question remained open as to how far the lower density IGM (causing the much more frequent, unsaturated Ly $\alpha$ forest systems) has been affected. In the present paper, we use the Keck High Resolution Echelle Spectrometer (HIRES; Vogt et al. 1994) to investigate the fabric of the $L y \alpha$ forest proper on subkiloparsec scales at high signal-to-noise ratio $(\mathrm{S} / \mathrm{N})$ and with a velocity resolution sufficient to resolve the width of the Lyman lines and detect velocity gradients of a few hundred meters per second.

The most suitable object known for this sort of study is the ultrabright, quadruple, gravitationally lensed QSO $\mathrm{Q} 1422+231\left(z_{\mathrm{em}}=3.61\right.$; Patnaik et al. 1992). This object has been observed for similar purposes previously at low resolution. Bechtold \& Yee (1995) concluded from ground- based spectra that there are differences of typically about $50 \%$ in $\mathrm{H}$ I column density for beam separations on the order of $100 \mathrm{pc}$. In contrast, Petry et al. (1998), from Hubble Space Telescope Faint Object Spectrograph spectra, found very little difference in both column density and velocity between the various lines of sight. In $\S 2$ we describe Keck HIRES (Vogt et al. 1994) spectra of the A and C images of $\mathrm{Q} 1422+231$. The properties of this lens system are summarized on the CASTLES Web site. ${ }^{5}$ The observations of images A and C of Q1422+231 and the methods used to reduce the data are discussed in $\S 2$. Section 3 contains an account of the measurement of the differences in the Ly $\alpha$ lines in the spectra of the two images. Our conclusions are summarized in $\S 4$.

\section{OBSERVATIONS AND DATA ANALYSIS}

Q1422 + 231 consists of four images. Images A $(g=16.92$ mag), B (16.77 mag), and C (17.44 mag) are almost along a line with $C$ separated by 1".64 from A and 0.76 from B. Image D (20.56 mag) is well separated from the rest. We chose to obtain HIRES spectra of A and C, using a 0".56 slit and only observing in conditions of excellent seeing. Much of the guiding was done manually, and the position angle of the slit was chosen so as to keep image B out of the slit. In some exposures we guided on the outer edge of the image in order to ensure minimal contamination from B. The data set is the same as described in Rauch, Sargent, \& Barlow (1999, hereafter Paper I) and Paper II. The spectra were reduced as described in Barlow \& Sargent (1997). The individual $\mathrm{A}$ and $\mathrm{C}$ exposures were matched in flux to a template produced by adding up all A spectra, using polynomial fits typically of 8 th order per echelle order. Care was taken to include only points with flux levels close to the continuum in the fitting regions and to avoid absorption lines. This way, the large-scale features in the spectra become identical (the information on large-scale differences between the QSO continua is lost), but features on scales up to $\sim 300-400 \mathrm{~km} \mathrm{~s}^{-1}$ are retained. Thus, differences among individual absorption line profiles up to that velocity scale survive our matching procedure and remain unaffected. The resulting spectra were normalized to a unit continuum using spline functions. Voigt profiles were fitted to the absorption lines using the fitting routine VPFIT ${ }^{6}$ (Carswell et al. 1991), until the fit exceeded a minimum probability $(1 \%)$ to produce by chance a $\chi^{2}$ as high as the one attained. We departed here from previous such analyses in that the whole spectrum was fitted (in continuous pieces), without selecting significant absorption features by eye. We thus do not have to assign significance thresholds to individual lines, a procedure that is most meaningful for single unblended, strong absorption components but which becomes uncertain in more complicated situations. Another problem, peculiar to relatively high $\mathrm{S} / \mathrm{N}$ spectra such as those obtained with HIRES, is that it is hard to obtain satisfactory fits merely by superimposing Voigt profiles, even if Voigt profiles are a good physical model. When $\mathrm{S} / \mathrm{N}$ ratios exceed a few tens, the errors in the fit are invariably dominated by systematic uncertainties in the placement of the continuum level. Therefore, it was decided to treat the continuum in every fitting region (average length $28 \AA$ ) as a

\footnotetext{
${ }^{5}$ See http://cfa-www.harvard.edu/glensdata/B1422.html.

${ }^{6}$ R. F. Carswell, J. K. Webb, A. J. Cooke, \& M. J. Irwin 1992, Institute of Astronomy, Cambridge; http://www.ast.cam.ac.uk/ rfc/vpfit.html.
} 
free parameter. This resulted in a mean drop of the continuum level over the region $4747-5630 \AA$ (i.e., between $\operatorname{Ly} \beta$ and $\operatorname{Ly} \alpha$ emission, where the $\operatorname{Ly} \alpha$ analysis in the present paper was performed) to $99.3 \%$ of the original level, with a standard deviation of $2.7 \%$ of the original continuum level; i.e., the total continuum level remained virtually unaffected by introducing locally free continua, but the local continua were adjusted typically by $2.7 \%$ up or down to obtain an optimal fit.

Since the A and C images are so close to B, we have to be concerned that blending of the spectrum of an image with light from the other images might have reduced the differences seen between the spectra. However, our spectra show strong column density differences between the $\mathrm{A}$ and $\mathrm{C}$ images in some low-ionization metal lines in the Ly $\alpha$ forest (see Paper I), which would have been significantly reduced if spillover of light from different images had been a serious problem. ${ }^{7}$ Our technique of matching the two spectra to a common template as described earlier does take out the large-scale fluctuations but not those among individual lines. Again, the differences seen in metal line systems suggest that this is not a problem. Finally, while they may affect the measured column densities, none of these effects could conspire to reduce any actual intrinsic velocity differences between the lines of sight to values as tiny as those found here.

The lowest redshift covered by the selected spectral region, $z=2.905$, corresponds to a transverse separation $\Delta r \sim 270$ pc. Throughout the paper beam separations are computed for $H_{0}=50 \mathrm{~km} \mathrm{~s}^{-1} \mathrm{Mpc}^{-1}$ and $q_{0}=0.5$. The redshift of the lens is taken to be $z=0.338$ (Kundic et al. 1997).

\section{DIFFERENCES BETWEEN THE LINES OF SIGHT IN THE Ly $\alpha$ FOREST REGION}

Here we describe three main approaches to measuring differences between the Ly $\alpha$ forests in the two adjacent lines of sight: a global cross-correlation analysis; a search for column density and velocity differences among individual absorption lines; and a pixel-by-pixel measurement of relative fluctuations in the optical depth between the lines of sight.

\subsection{Global Correlation Analysis}

We can study global differences in the Ly $\alpha$ forest region by measuring the cross-correlation function, $\xi_{\mathrm{cc}}$, over the total, useable length of both spectra. We define this quantity by

$$
\begin{aligned}
& \xi_{\mathrm{cc}}(\Delta v, \Delta r) \\
& \equiv \frac{\left\langle\left[F_{r}(v)-\left\langle F_{r}\right\rangle\right]\left[F_{r+\Delta r}(v+\Delta v)-\left\langle F_{r+\Delta r}\right\rangle\right]\right\rangle}{\sqrt{\left\langle\left[F_{r}(v)-\left\langle F_{r}\right\rangle\right]^{2}\right\rangle\left\langle\left[F_{r+\Delta r}(v+\Delta v)-\left\langle F_{r+\Delta r}\right\rangle\right]^{2}\right\rangle}} .
\end{aligned}
$$

Here $F_{r}$ and $F_{r+\Delta r}$ are the pixel flux values of the two spectra, separated by $\Delta r$ on the plane of the sky. The velocity coordinate along the line of sight is $v$ (where $d v=c d \lambda / \lambda$ ),

\footnotetext{
${ }^{7}$ We have also obtained as yet unpublished spectra of the lensed QSO UM673 with HIRES. In this case the images A and B have a separation of 2 ".2 so there is little risk of mixing the light from different images. The spectra have significantly lower $\mathrm{S} / \mathrm{N}$ than the data on Q1422+231 discussed here, but the absence of differences between the low column density Ly $\alpha$ forest lines in UM 673A, B is consistent within the errors with the very small differences found in the present paper.
}

and $\Delta v$ is the velocity lag. The averages are taken over the whole velocity extent of the spectrum. For $\Delta r=0$ we get the usual autocorrelation function $\xi_{\mathrm{cc}}(\Delta v, 0)$, while for $\Delta v=0$ we have the cross-correlation as a function of transverse separation only. The function is defined so as to satisfy $\xi_{\mathrm{cc}}(0,0)=1$. With large-scale velocity correlations $(>1000$ $\mathrm{km} \mathrm{s}^{-1}$ ) expected to be absent or weak (Sargent et al. 1980), the autocorrelation function (on scales $\sim 100 \mathrm{~km} \mathrm{~s}^{-1}$ ) mostly measures the Ly $\alpha$ line width and the weak smallscale clustering of $\operatorname{Ly} \alpha$ forest systems (e.g., Webb 1987; Rauch et al. 1992). We apply the correlation analysis to most of the spectral region between $\operatorname{Ly} \beta$ and $\operatorname{Ly} \alpha$ emission, 4737-5630 A. Only regions 4747-4875, 4895-4997, 5003$5221,5227-5409,5419-5473$, and 5480-5630 ̊ were used in the analysis in order to avoid contamination by known metal systems. These regions are shown in Figure 1; the hatched parts were excluded. The resulting mean redshift $\bar{z}=3.26036$ of the remaining sample corresponds to a mean beam separation $\overline{\Delta r}\left(=119 h_{50}^{-1} \mathrm{pc}\right)$. A small portion of the spectrum is shown in Figure 2. The spectrum of image C (dotted line) is overplotted on that of A (solid line) to illustrate how closely the spectra resemble each other.

The function $\xi_{\mathrm{cc}}(\Delta v, \overline{\Delta r})$ is shown in Figure 3. In particular, we obtain the "zero-lag" cross-correlation function

$$
\xi_{\mathrm{cc}}\left(\Delta v=0 ; \overline{\Delta r}=119 h_{50}^{-1} \mathrm{pc}\right)=99.48 \% .
$$

Thus, over a mean transverse distance of $120 \mathrm{pc}$ the appearance of the Ly $\alpha$ forest changes very little, and even the small difference observed may be because of noise or systematic errors.

\subsection{Comparison between Individual Absorption Lines}

We next investigate how properties of individual absorption systems change with the separation on the plane of the sky. We can quantify differences $\Delta x=x_{1}-x_{2}$ in any physical property $x$ of absorption systems common to both lines of sight 1 and 2 by means of the distribution function of $\Delta x$. (For example, consider the difference between the

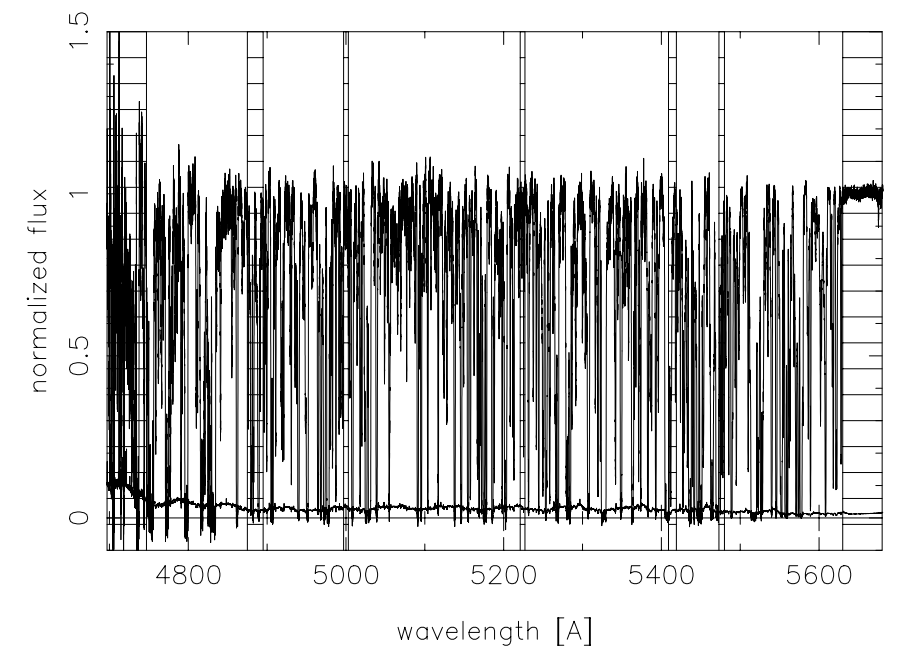

FIG. 1.-Normalized Ly $\alpha$ forest spectra of the QSO $1422+231$ A image in the wavelength region between $\operatorname{Ly} \beta$ and $\operatorname{Ly} \alpha$ emission. The shaded areas were excluded from the cross-correlation analysis as not belonging to the forest or as being contaminated by known metal absorption lines. The usable Ly $\alpha$ forest region extends over the wavelength range $4747-5630 \AA$ or redshift range $z=[2.90,3.63]$. The maximum beam separation at the short wavelength end of the spectrum is $270 h_{50}^{-1} \mathrm{pc}$. The slowly varying function at the bottom is the $1 \sigma$ error. 


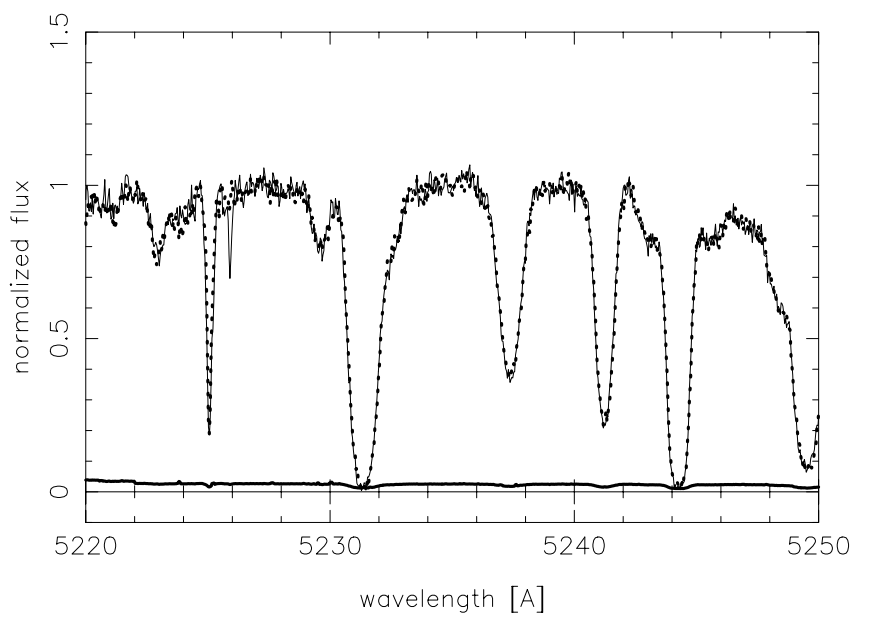

FIG. 2.-Enlargement of part of the spectrum of Q1422+231. The spectra of the A (solid line) and C (dotted line) images are plotted on top of each other. There is hardly any difference between the spectra, with the exception of a narrow line (which is largely absent in the spectrum of the $\mathrm{C}$ image) and some lower column density fluctuations near $5226 \AA$. This line and the sharp stronger one at $5225 \AA$ can be identified with an Si IV $\lambda 1394$ interloper from an absorption system at $z=2.74889$. The other absorption features are plausibly attributed to $\mathrm{H}$ I $\mathrm{Ly} \alpha$. The slowly varying function plotted at the bottom is the $1 \sigma$ error of image A.

redshifts of the lines $z_{1}-z_{2}$ ). In an isotropic universe, the mean, $\overline{\Delta x}$, vanishes, so the information is contained in the shape of the distribution of differences, as characterized, for example, by the observed scatter of the distribution, $\sigma(\Delta x)$. The width of the intrinsic distribution, $\sigma_{\text {int }}(\Delta x)$, differs from the observed one owing to the combined measurement errors, $\sigma\left(x_{1}\right)$ and $\sigma\left(x_{2}\right)$, and any systematic error $\sigma_{\text {sys }}$ resulting from the occasional erroneous pairing of two unrelated absorption components. The quantity $\sigma_{<}(\Delta x)=$ $\left[\sigma(\Delta x)^{2}-\sigma\left(x_{1}\right)^{2}-\sigma\left(x_{2}\right)^{2}\right]^{1 / 2}$ is then a strict upper limit on the intrinsic scatter, $\sigma_{\text {int }}(\Delta x)$.

Here we concentrate on the distribution of velocity and column density differences, $\Delta v$ and $\Delta \log N$. Inspection by eye shows that there are only minute difference between the spectra of the A and C images. Accordingly, it is legitimate to associate each $\mathrm{Ly} \alpha$ forest line in the spectrum of component $\mathrm{A}$ with a corresponding line in $\mathrm{C}$. Thus, after profile-

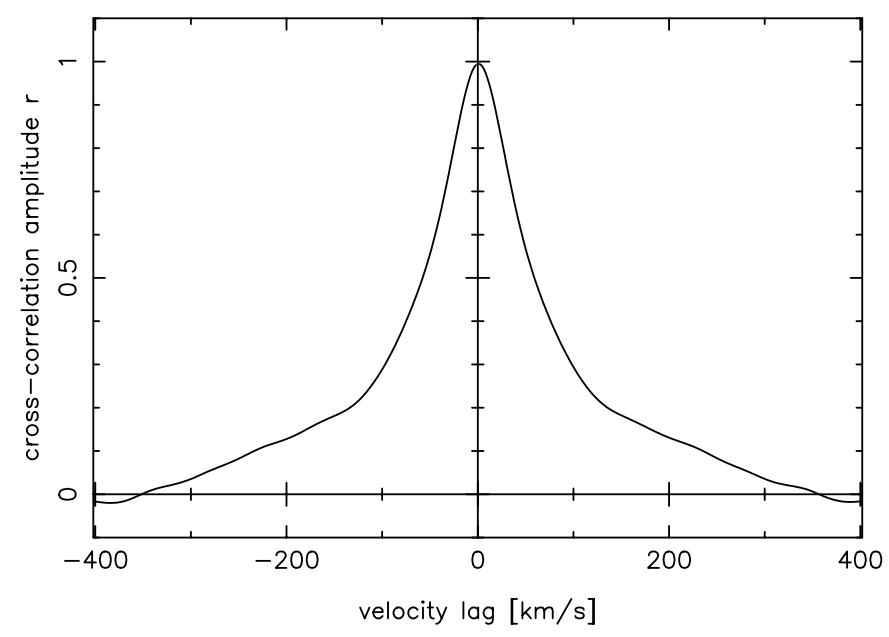

FIG. 3.-Cross-correlation function $r(\langle d\rangle, \Delta v)$, for a mean beam separation $\langle d\rangle=119 h_{50}^{-1} p c$ between the Ly $\alpha$ forests in the Q1422+231 A and $\mathrm{C}$ image, for the spectral ranges shown in Fig. 1. fitting the spectra, we suppose that an absorption component in spectrum $\mathrm{C}$ belongs to the same underlying physical structure as a line in A if its redshift and column density fall within a given redshift and column density window centered on a similar component in the A spectrum.

We make the assignments totally automatically in order to preclude human prejudice. In order to avoid too many random associations, we restrict the allowed range of column density differences when measuring the relative velocity distribution and restrict the allowed range of velocity differences when comparing the column densities. Specifically, the redshifts had to agree to within $\pm 30 \mathrm{~km} \mathrm{~s}^{-1}$ (there is no real prejudice as this is much larger than the actual scatter), while column density differences $\left|\log N_{A}-\log N_{C}\right|<1$ were deemed acceptable. In order to avoid including ill-constrained measurements and spurious cross-identifications, all lines included were required to have relative Doppler parameter errors, $\sigma(b) / b<3$ and column density errors $\sigma(\log N) / \log N<3$. Doppler parameter values had to fall in the interval $5-300 \mathrm{~km} \mathrm{~s}^{-1}$ to exclude spuriously low values owing to noise spikes and high values because of unresolved blends. All error estimates given below are $1 \sigma$ deviations derived from the output of the line profile fitting program. Finally, to get rid of obvious misassociations, one iteration of a $4 \sigma$ clipping algorithm was performed. Column density differences larger than 4 standard deviations of the mean of the distribution of column density differences where rejected. The analysis was limited to column densities $12<\log N<14.13$, the lower bound being close to the detection limit, whereas the upper limit (14.13) corresponds to a residual flux of about $2.4 \%$ (for a Doppler parameter of $28 \mathrm{~km} \mathrm{~s}^{-1}$, typical of an unsaturated $z \sim 3 \mathrm{Ly} \alpha$ forest line), a value just at the top of the linear part of the curve of growth but far enough away from saturation to permit us to see significant differences between the central optical depths. This column density regime is most relevant for the study of gravitational structure formation since the selected absorption lines probe gas overdensities still close to the linear regime of gravitational instability $(\Delta \rho / \rho \leq 15)$ on the scale of individual galaxies (e.g., Miralda-Escudé et al. 1996).

\subsubsection{Velocity Differences}

Figure 4 shows the observed histogram of velocity differences, $\Delta v=v_{A}-v_{C}{ }^{8}$ The top plot of Figure 5 shows the observed standard deviations of the distribution of velocity differences between the two lines of sight (filled circles) versus the largest measurement error in redshift for lines remaining in the sample. The open diamonds show the predicted width of the distribution of velocity differences expected from statistical measurement errors alone. It was

\footnotetext{
${ }^{8}$ The original distribution did not have a zero mean but showed a mean shift of spectrum $\mathrm{C}$ by $0.96 \mathrm{~km} \mathrm{~s}^{-1}$. This shift amounts to slightly less than half a pixel or a quarter of the slit width. Its size and the circumstances of the observations make it most likely that it was caused by systematic variations in the position of the target on the slit: the targets were only observed in exceptional seeing to minimize contamination of the desired image by the light from other images. Consequently, the seeing may often have been better than the 0.574 slit width, leading to a dependence of the wavelength zero point on the actual position of the target in the slit. This uncertainty may have been turned into a systematic shift by our attempts to avoid spillover. For the analysis below and for all the plots from Fig. 4 on, the above shift was subtracted from the velocities of the C spectrum.
} 


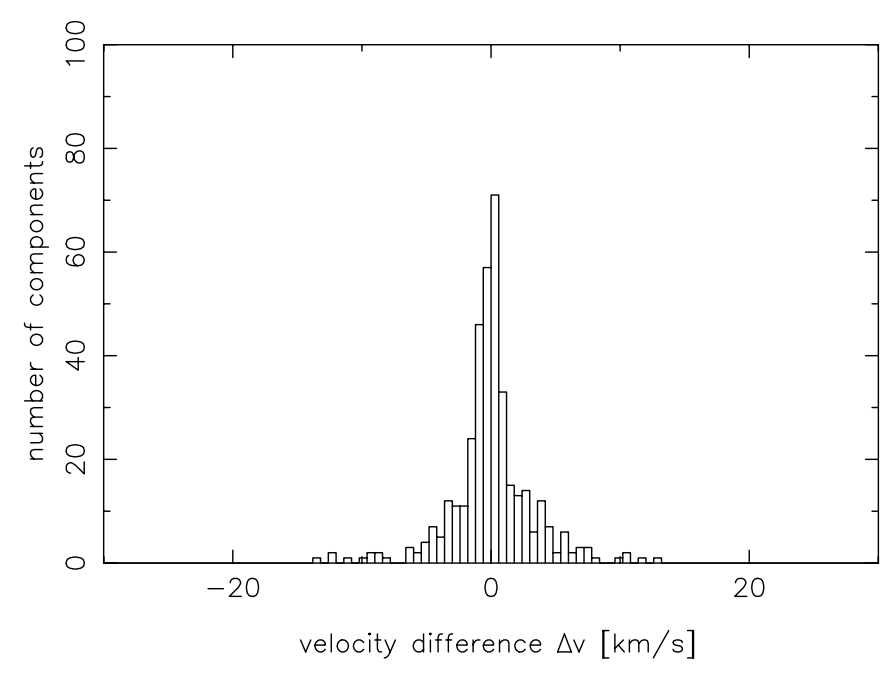

FIG. 4.-Distribution of the velocity differences $\Delta v\left(=v_{A}-v_{C}\right)$ for pairs of absorption components. Two components were considered to correspond to each other, when they were nearest neighbors, lay within $30 \mathrm{~km}$ $\mathrm{s}^{-1}$ of each other, and had column densities differing by less than $50 \%$.

computed as the quadratic sum of the $1 \sigma$ error estimates produced by the Voigt profile fitting routine. To search for intrinsic scatter we discard those velocity differences with large errors. As the most uncertain points are eliminated, the observed width of the distribution should decrease, and any intrinsic scatter should emerge. We see that the farther one goes to the left along the abscissa, the line pairs remaining in the sample have smaller and smaller maximum (and mean) redshift errors and the more well defined are the measurements. In other words, if the bulk of the scatter is caused by measurement error, we should expect to see the observed total scatter $\sigma(\Delta v)$ decreasing in step with, and closely tracking, the measurement errors as increasingly stringent error limits are applied and more and more data points are discarded from the distribution. Aside from a certain scatter (probably caused by mismatched components), this behavior is indeed observed in Figure 5. (The discrepancy at large values of the abscissa between the width of the predicted distribution based on measurement errors alone and the observed distribution is an artifact of the range of parameters we imposed earlier. It is caused by our admitting only velocity differences less than $30 \mathrm{~km} \mathrm{~s}^{-1}$ into the sample. This is perfectly justified given the range of the large majority of values in Figure 4, but it does get rid of some large outliers that result from automatic misidentifications of line pairs.)

To demonstrate the sensitivity of this method, we plot (for illustrative purposes) the observed $\sigma(\Delta v)$ a second time (Fig. 5, upper panel, dashed line), but now with an additional random, Gaussian velocity jitter of $\sigma=1.5 \mathrm{~km} \mathrm{~s}^{-1}$ imposed on each line pair; i.e., the distribution of pair velocity differences now has a width of $\left[\sigma^{2}(\Delta v)+\left(1.5 \mathrm{~km} \mathrm{~s}^{-1}\right)^{2}\right]^{1 / 2}$. On the left half of the diagram this jitter clearly dominates the observed width, demonstrating that any intrinsic velocity differences of that size would have been seen easily and that any actual differences must be considerably less than $1.5 \mathrm{~km}$ $\mathrm{s}^{-1}$.

There are 259 line pairs with individual redshift errors $\sigma(z)<4 \times 10^{-5}$ with an observed total $\sigma(\Delta v)=1.7 \mathrm{~km} \mathrm{~s}^{-1}$, and still 83 pairs in the bin with the smallest errors, $\sigma(z)<1 \times 10^{-5}\left(\sigma(\Delta v)=0.6 \mathrm{~km} \mathrm{~s}^{-1}\right)$. The upper limits
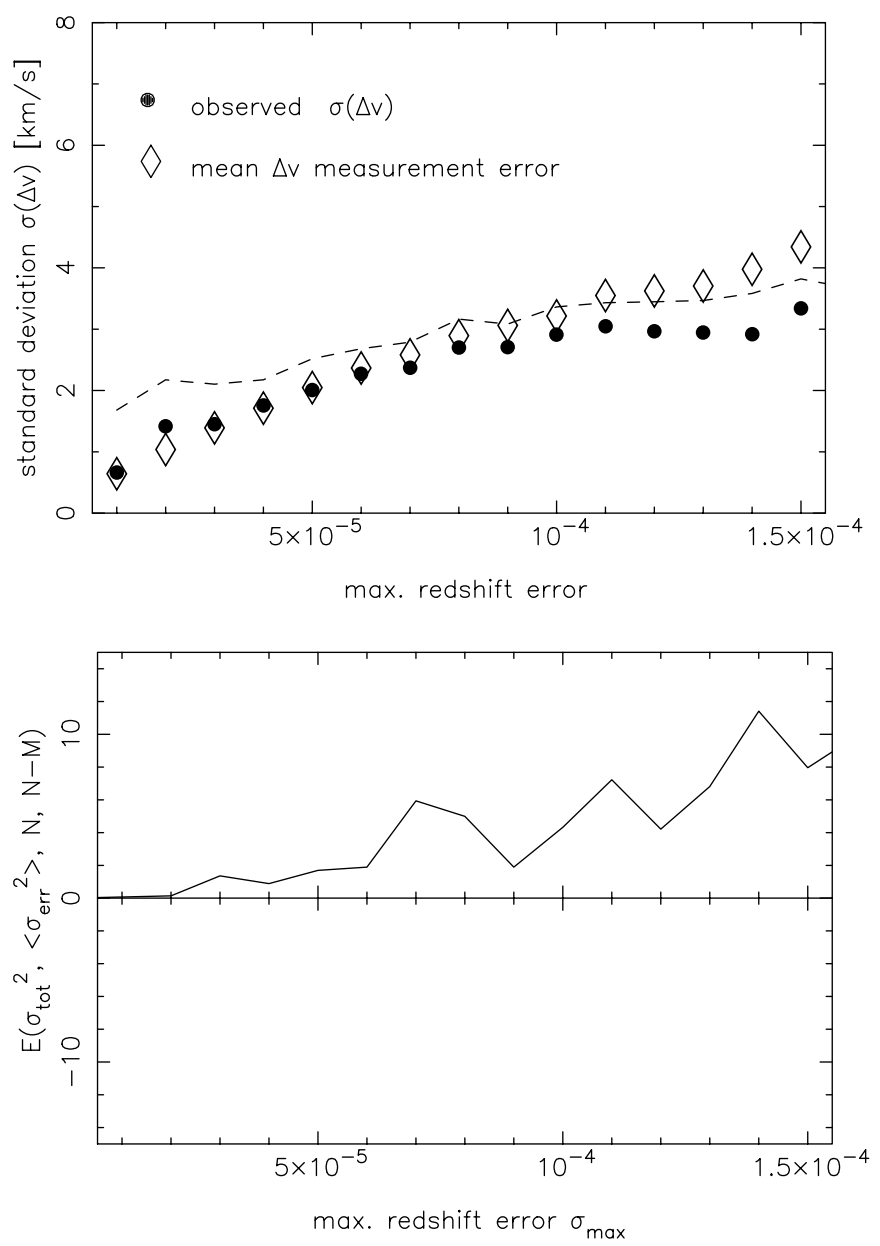

Fig. 5.-Top: rms difference between the velocities of individually fitted absorption components in the two lines of sight vs. the maximum measurement error permitted for the redshifts in the sample used. Filled circles give the observed width of the $\Delta v$ distribution, open diamonds the width expected on the basis of statistical errors in the velocity determination alone. The dashed line shows the width of the observed distribution, convolved with a Gaussian scatter with $\sigma=1.5 \mathrm{~km} \mathrm{~s}^{-1}$. Bottom: Function $E$ giving the merit of increasingly getting rid of the data points with the largest error (see also Fig. 8). The value of $E$ shows the trade-off between omitting measurement points with errors above a certain value and the consequent reduction in the size of the sample vs. the maximum measurement error accepted in the sample. Positive values of $E$ imply that it is beneficial to continue throwing away points with the largest errors; negative values imply a loss of information. The zero crossing corresponds to the optimum choice. The meaning of $E$ is explained in the text and the Appendix.

$\sigma_{<}(\Delta v)$ on the intrinsic $\sigma_{\text {int }}(\Delta v)$ (where the measurement errors have been subtracted in quadrature from the observed width) are formally 0.4 and $0.2 \mathrm{~km} \mathrm{~s}^{-1}$, respectively. Given the scatter between the measurement errors and total $\sigma(\Delta v)$ visible in the plot, the intrinsic distribution is probably consistent with zero width. We can compare these values with the velocity differences expected from the Hubble expansion alone. If Ly $\alpha$ clouds expand with the Hubble flow, the projected velocity differences between two lines of sight (modeling the geometry by an expanding slab intersecting both lines of sight at an angle $\alpha$ ) would be on the order of $\Delta v_{\mathrm{H}} / \tan \alpha=H(\langle z\rangle) \quad r \sim 50 \mathrm{~ms}^{-1}$, where $H(\langle z\rangle=3.26)=440 h_{50} \mathrm{~km} \mathrm{~s}^{-1} \mathrm{Mpc}^{-1}$ was adopted as the Hubble constant at the mean observed redshift. This is well below our detection threshold, and our null result is consistent with the clouds following the Hubble flow. 
It is tempting to try using the measurement of $\sigma(\Delta v)$ to get a rough idea of the energy deposited in the gas in the form of turbulence. In Paper II we had applied the Kolmogorov relation between the structure function $B(r)$, and the energy input rate $\epsilon$ at scale $r$,

$$
B(r) \approx \overline{\left[v_{1}\left(r^{\prime}\right)-v_{2}\left(r^{\prime \prime}\right)\right]^{2}} \approx(\epsilon r)^{2 / 3}
$$

(e.g., Kaplan \& Pikelner 1970; here $v_{1}\left(r^{\prime}\right)$ and $v_{2}\left(r^{\prime \prime}\right)$ are the velocities along the line of sight, $r$ is the spatial separation between the lines of sight, and the average is taken over all absorption line pairs with mean transverse separation $r$ ) to get an approximate estimate of the energy input rate $\epsilon$ in C IV absorbing clouds. These gas clouds correspond to strong, usually saturated Ly $\alpha$ forest systems and overdensities higher than a few tens. This approach could be justified then as we had indeed observed turbulent energy on a wide range of scales and had found a rise of $B(r)$ with $r$; moreover, the relevant timescales for energy dissipation were short, so turbulent energy must have been propagating down over a range of scales close to the epoch of observation. Now we are applying this scheme to the lower density gas giving rise to unsaturated low column density Ly $\alpha$ lines, which has densities within a factor of 10-15 of the mean density of the universe, and, at average, must be situated much farther away from sources of stellar energy. Here it is more likely that any energy injection by stellar feedback is intermittent or happened only once long ago, so a steady state (one of the conditions for the validity of the Kolmogorov law) may not exist (or no longer exists).

With this warning in mind we proceed to calculate $\epsilon$ for our present low column density Ly $\alpha$ forest sample. The quantity $\epsilon$ is still a measure of the energy flow on a given scale but is not necessarily related to the current primary energy input on larger scales (e.g., by explosions or winds). If we identify our measured variance $\sigma^{2}(\Delta v)$ with $B(r)$, we can solve for the energy transfer rate ${ }^{9}$ at scale $r$ :

$$
\epsilon \sim \frac{\sigma^{3}(\Delta v)}{r}
$$

Adopting the above value $\sigma_{\text {int }}(\Delta v)=0.4 \mathrm{~km} \mathrm{~s}^{-1}$ and $\bar{r}=110 h_{50}^{-1} \mathrm{pc}$,

$$
\epsilon \sim 2 \times 10^{-7} h_{50} \operatorname{ergs~g}^{-1} \mathrm{~s}^{-1} .
$$

Thus, the IGM giving rise to the unsaturated parts (i.e., most) of the $\mathrm{Ly} \alpha$ forest at $z=3.26$ is experiencing energy transfer at a rate 3-4 orders of magnitude less than the gas giving rise to the $\mathrm{C}$ IV absorption systems dealt with in Paper II, ${ }^{10}$ and 6-7 orders of magnitude less than the values in the Orion nebula, for example.

\subsubsection{Column Density Differences}

A scatter plot of the column densities in $\mathrm{A}$ and $\mathrm{C}$ is given in Figure 6. The scatter is larger in the $\mathrm{C}$ image because of the lower $\mathrm{S} / \mathrm{N}$ there. Some of the outliers may come from the ambiguity of the Voigt profile models (which occasionally may lead to similar absorption complexes

\footnotetext{
${ }^{9}$ Because of the uncertain geometry of the clouds and our taking the known projected transverse separation instead of the (unknown) actual three-dimensional separation between points (see the discussion in paper II), this approach can provide only a rough, order of magnitude estimate of $\epsilon$.

${ }^{10}$ But note that the $\mathrm{C}$ IV turbulence was measured on a 3 times larger scale, $300 \mathrm{pc}$, so the discrepancy may be less.
}

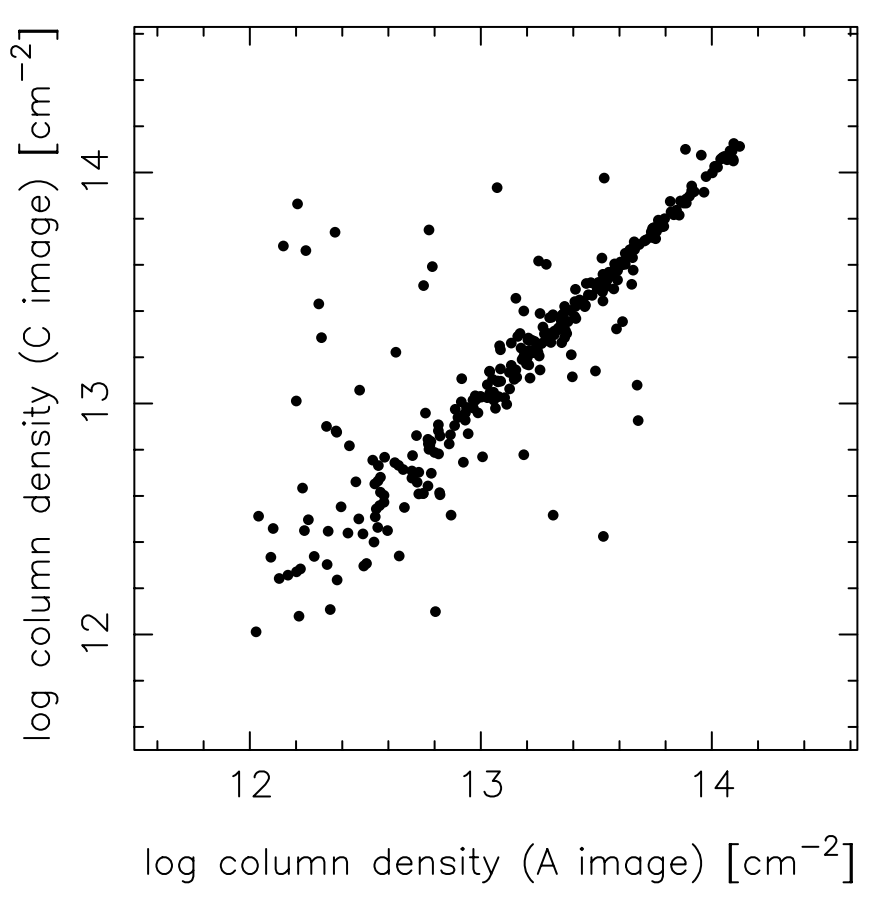

FIG. 6.-Scatter diagram of the logarithmic column densities in the A and $\mathrm{C}$ spectrum

being modeled automatically by two rather different sets of Voigt profile components), while others may be genuine differences owing to unidentified metal lines. The distribution of column density differences is shown in Figure 7. Two components were considered to correspond to each other, when they were nearest neighbors, lay within $30 \mathrm{~km} \mathrm{~s}^{-1}$ of each other, and had column densities differing by less than 1.0 dex. Figure 8 shows the scatter versus measurement error diagram for column densities, similar to Figure 5. For the best-defined samples (those with measurement errors around $5 \times 10^{-2}$ in $\log N$ ), the corrected upper limit to the intrinsic scatter is $\sigma_{<}(\Delta \log N) \sim 2 \times 10^{-2}$. We note that it makes sense to discuss an "optimum sample" because discarding those data points with the largest measurement

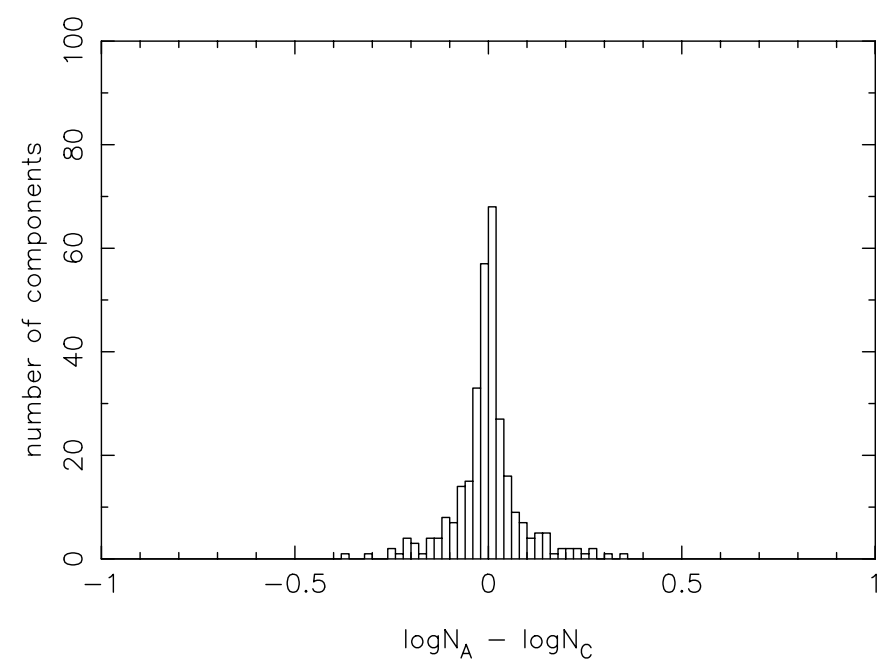

FIG. 7.-Distribution of the logarithmic column differences $\Delta \log N\left(=\log N_{A}-\log N_{C}\right)$ for pairs of absorption components. Two components were considered to correspond to each other when they were nearest neighbors, lay within $30 \mathrm{~km} \mathrm{~s}^{-1}$ of each other, and had logarithmic column densities differing by less than 1.0 . 

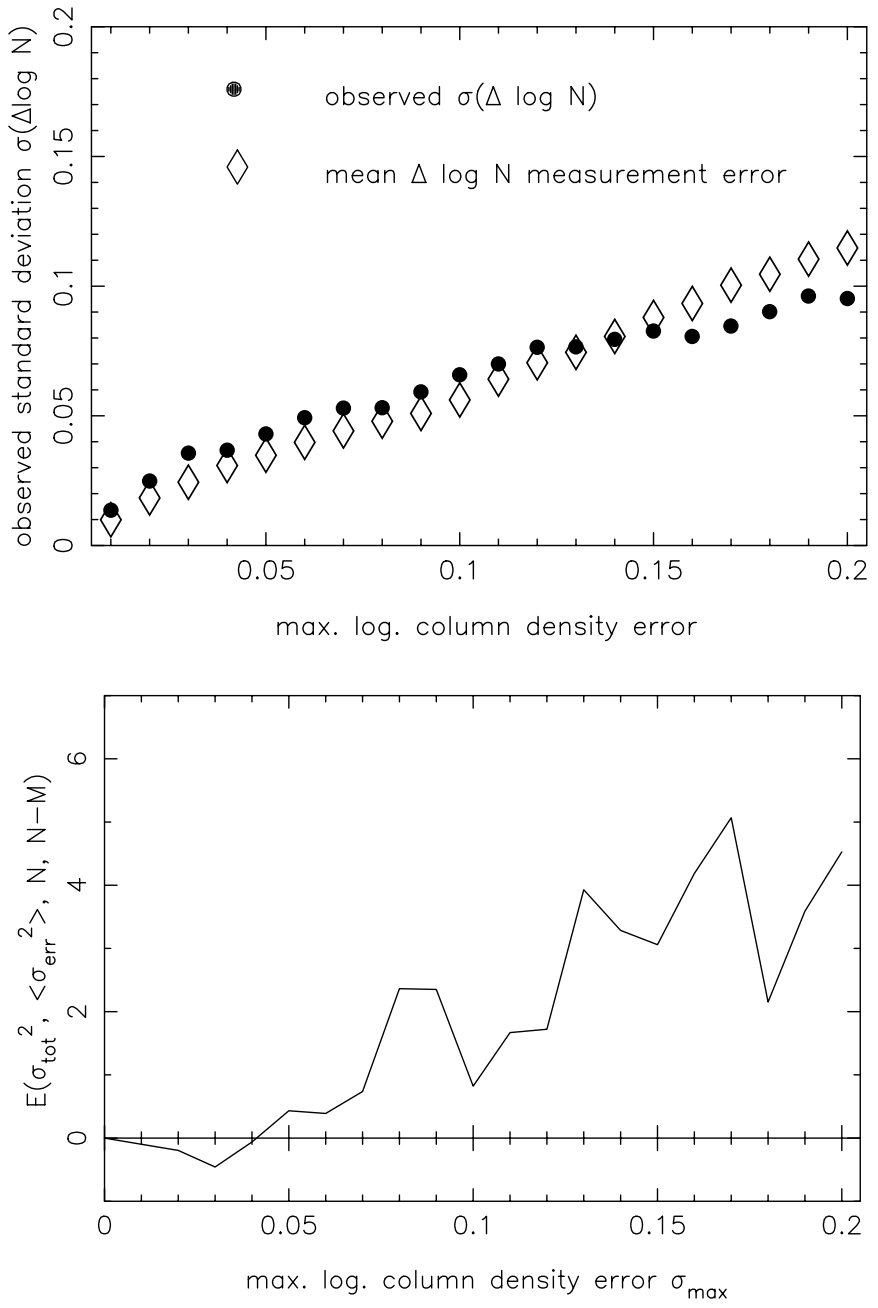

FIG. 8.-Top: rms difference between the column densities of individually fitted absorption components in the two lines of sight vs. the maximum measurement error permitted for the column densities in the sample used. Filled circles give the observed width of the $\Delta \log N$ distribution, open diamonds the width expected on the basis of statistical errors in the column density determination alone. Bottom: The value of function $E$ shows the trade-off between omitting measurement points with errors above a certain value and the consequent reduction in the size of the sample vs. the maximum measurement error accepted in the sample. Positive values of $E$ imply that it is beneficial to continue throwing away points with the largest errors, negative values imply a loss of information. The zero crossing corresponds to the optimum choice.

error leaves better measurements but also reduces the sample size and thus the statistical significance. Initially throwing away the points with the worst error will improve the precision with which sample mean and variance are known, but the precision of these statistical values decreases with the square root of the number of data points, $\mathscr{N}^{1 / 2}$ and if enough points have been lost the penalty gets larger than the gain. In Appendix A we have defined a function of merit, $E$, which is positive as long as it is beneficial to discard data points, and crosses zero toward negative values if the sample becomes to small. The bottom panels of Figures 5 and 8 show this function. For example, from the column density case (Fig. 8) we find that the sample has been cut down to optimal size by retaining only data points with a maximum column density error $\leq 0.05$.

Most interesting from an astrophysical point of view is whether we can put limits on the total baryon density fluc- tuations on the scale of the transverse separations of the two lines of sight. The relation between the $\mathrm{H}$ I column density $N(\mathrm{H} \mathrm{I})$ and the baryon density for Jeans-smoothed IGM regions can be written as $N(\mathrm{H} \mathrm{I}) \propto \rho_{\text {bar }}^{\alpha}$ with $\alpha=1.37$ 1.5 (Schaye 2001). For this power law the distribution of column density differences $\Delta \log N\left(=\log N_{A}-\log N_{B}\right)$ can be transformed by simple scaling with $1 / \alpha=0.73$ (we use the smaller value $\alpha=1.37$ to establish the most conservative upper limit) into a distribution of density differences, and the variance of the logarithmic baryon density becomes

$$
\left\langle(\Delta \log \rho)^{2}\right\rangle \leq \alpha^{-2}\left\langle\left(\log N_{A}-\log N_{B}\right)^{2}\right\rangle .
$$

Adopting (conservatively) the pair sample with mean column density measurement errors below 0.05 dex (153 lines; see Fig. 8), we arrive at a value of $2.5 \times 10^{-2}$ for the corrected "intrinsic" scatter and $4.3 \times 10^{-2}$ for the uncorrected, observed scatter in $\Delta \log N$. As the figures show, other choices of sample size give similar values. Then we get

$$
\sqrt{\left\langle(\Delta \log \rho)^{2}\right\rangle} \leq 1.8 \times 10^{-2} \text { (or } 3.1 \times 10^{-2} \text {, uncorrected) }
$$

for the typical logarithmic change in density on a scale of $0.110 h_{50}^{-1} \mathrm{kpc}$; i.e., the rms fluctuation in the baryon density is less than about $3.1 \%$. From the close agreement between the width of the observed distribution and the expected width from measurement errors alone, it is clear that the fluctuations are dominated by the measurement errors and that the intrinsic fluctuations are consistent with being zero at our level of precision.

\subsection{The Filling Factor of "Disturbed" Regions in the Universe}

Here we address the question as to how much of the universe (by volume) is hydrodynamically disturbed, for example by supernova explosions or galactic winds along the two lines of sight. We consider a region in space at a given redshift $z$ as "disturbed" if the optical depth $\tau$ for H I Ly $\alpha$ absorption differs by more than a certain amount between the two lines of sight.

This is easy to measure unless the lines are saturated. Many of the saturated systems are associated with multicomponent metal absorption systems. These systems exhibit substantial variations in the absorption pattern across multiple, close lines of sight (e.g., Lopez et al. 1999; Paper II), but the component structure and any differences in the column densities are almost always completely

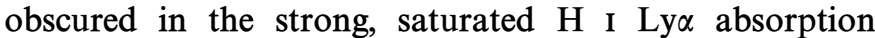
blends. Thus, to get a conservative upper limit on the fraction of disturbed regions we assume that saturated regions in the $L y \alpha$ forest are always disturbed; i.e., we treat them as if they had infinite optical depth differences.

Figure 9 shows the distribution of the difference in optical depth $\Delta \tau=\left|\tau_{A}-\tau_{C}\right|$ between the two lines of sight $A$ and $C$. The four different histograms show the results for various column density ranges ${ }^{11}$ (the column density in spectrum A was used to determine whether a pixel pair fell into a certain range): the solid line is for the whole spectrum, the dashed line for weak lines with $12.0<\log N<13.0$, the dashdotted line for $13.0<\log N<14.0$, and the dotted line for stronger, but still unsaturated, lines. (A column density

\footnotetext{
${ }^{11}$ The column densities quoted here are calculated by treating the observed optical depths as if they were central optical depths of Gaussian lines with Doppler parameter $28 \mathrm{~km} \mathrm{~s}^{-1}$.
} 


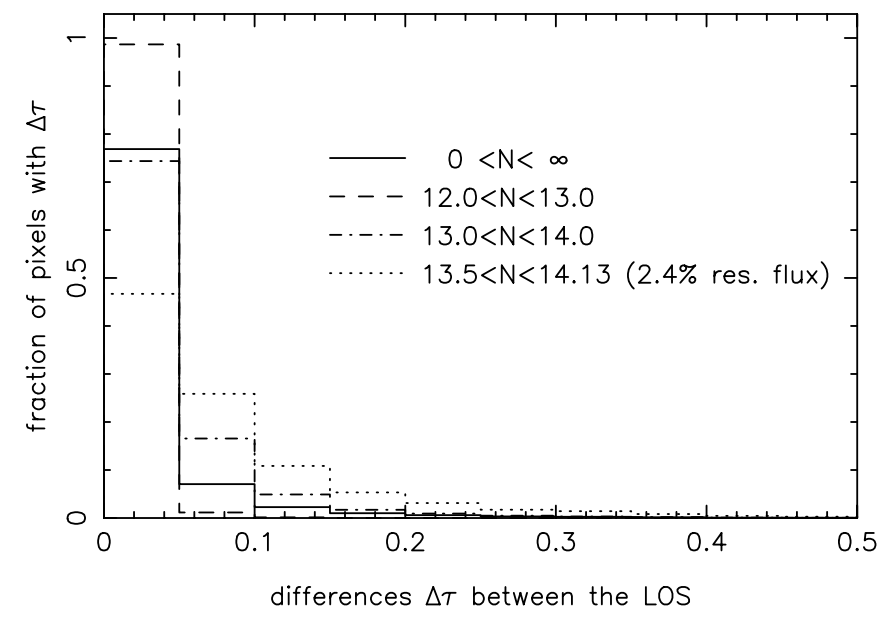

FIG. 9.-Distribution of differences between the H I optical depth $\Delta \tau=$ $\left|\tau_{A}-\tau_{C}\right|$ for various column density ranges. The fraction of pixels in the column density ranges shown are 100\% (solid line), 36.6\% (dashed line), $36.4 \%$ (dash-dotted line), and $17.4 \%$ (dotted line).

$\log N=14.13$ corresponds to a residual flux of $2.4 \%$ for the center of an absorption line with a typical Doppler parameter $b=28 \mathrm{~km} \mathrm{~s}^{-1}$ ). In the first histogram (solid line), saturated pixels (for practical purposes, those with $\log N>14.13$ ), did not provide any information and were assumed to have infinite optical depth differences $\Delta \tau$; thus they do not appear in this histogram in the lower bins. The relative proportions of the spectra in those four column ranges was $100 \%, 36.6 \%, 36.4 \%$, and $17.4 \%$, respectively. To reduce contributions to the optical depth differences from noise, both spectra were smoothed with a box filter on the scale of typical line broadening $\left(28 \mathrm{~km} \mathrm{~s}^{-1}\right)$.

We found that $76.8 \%, 98.7 \%, 74.7 \%$, and $46.7 \%$, respectively, of all the pixels in the four column bins had their optical depths differ between the two lines of sight by less than $\Delta \tau=0.05$ (i.e., they contribute only to the first bin of Fig. 9). It is remarkable that of the $36.6 \%$ of all pixels that are in the column density range $12.0<\log N<13.0,98.7 \%$ agree to within $\tau<0.05$. Apparently, the low column density $\operatorname{Ly} \alpha$ forest is virtually undisturbed by any effects capable of producing differences in density over a couple of hundred parsecs. The differences in optical depth increase with increasing column density as one would expect if higher column density gas is more closely associated with galaxies, which are the likely origin of any hydrodynamic disturbances.

The overall fraction of the Ly $\alpha$ forest with $\Delta \tau<0.05$ irrespective of column density is $1-f_{\text {LOS }}=76.8 \%$. Since we have counted regions close to or beyond saturation as having infinite $\Delta \tau$ (see above), $f_{\text {Los }}$ is a strict upper limit on the line-of-sight filling factor of the Ly $\alpha$ forest with absorption from processed gas capable of producing a disturbance of that magnitude.

\subsubsection{An Upper Limit to the Filling Factor of Galactic Wind Bubbles}

The upper limit $f_{\text {Los }} \leq 0.23$ on the fraction of disturbed pixels in the Ly $\alpha$ forest can be used in a crude way to estimate the number density and volume filling factor of galactic outflows from their effect on the density field of the IGM, insofar as such variations appear as differences between the $\mathrm{H}$ I column densities (Ly $\alpha$ forest opacities) between two close lines of sight. The principle will be illus- trated here for a model of wind bubbles expanding into the IGM (Fig. 10).

We assume that dense shells of swept-up material occasionally cross the double lines of sight to a lensed background QSO. It can be shown (see Appendix B) that even under unfavorable conditions the shells should have $\mathrm{H} \mathrm{I}$ column densities high enough to be seen in absorption in a high S/N QSO spectrum like the one of Q1422+231 discussed here $\left[N(\mathrm{H} \mathrm{I})>10^{12} \mathrm{~cm}^{-2}\right]$. The passage of the shell is very likely to disturb the resemblance between the absorption lines in the two lines of sight (Fig. 10). Ideally, the shell should produce two absorption systems at its two points of intersection with the lines of sight. We assume that there will be measurable differences in the absorption lines between the lines of sight as long as new shell material keeps crossing the lines of sight faster than the differences can be smoothed out by pressure waves traveling at the sound speed. Or in other words, a shell is no longer detectable for when the speed of sound $c$ exceeds the transverse velocity of the shell across the lines of sight:

$$
\frac{\dot{R}}{R} b<c,
$$

where $b$ is the impact parameter of the lines of sight relative to the center of the shell, and $R$ and $\dot{R}$ are the radius and expanding velocity of the shell.

To convert the line-of-sight filling factor into a volume filling factor, a particular model for the disturbance and its observational signature is needed. For illustrative purposes we adopt the supershell model of Mac Low \& McCray (1988); i.e., we assume that the disturbances are windblown shells escaping from high-redshift galaxies. For a given lineof-sight filling factor, the radius $R$, velocity of the shell $\dot{R}$, volume filling factor $f_{v}$, and the space density $n_{\text {gal }}$ of galaxies with a wind escaping into the IGM can be written in terms of the mechanical luminosity $L_{38}$ (in units of $10^{38} \mathrm{ergs} \mathrm{s}^{-1}$ ), the density of the ambient medium $n_{-5}$ (in units of $10^{-5}$

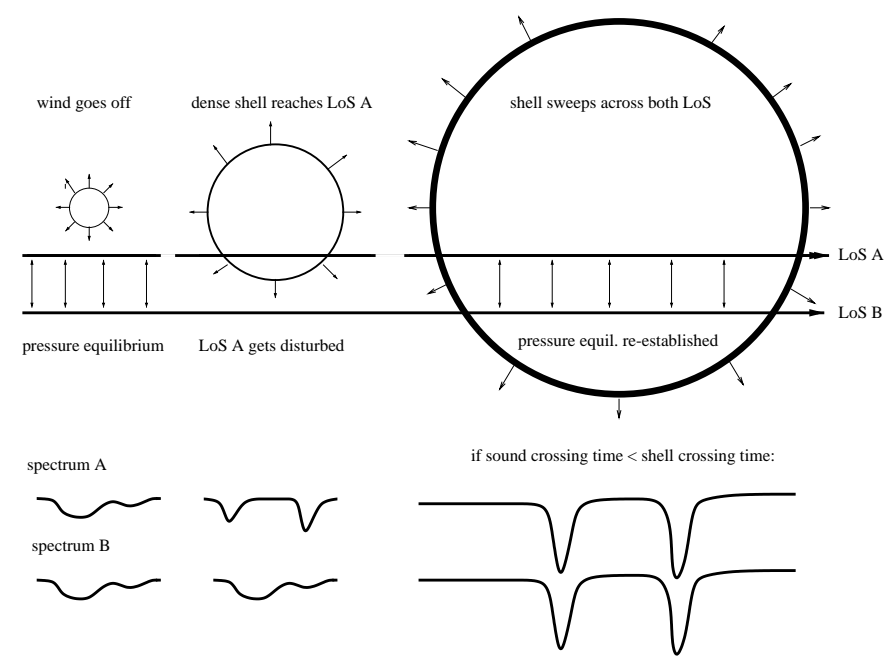

FIG. 10.-Left: Wind bubble expands near two lines of sight (A and B). The absorption spectra (bottom) look identical before the shell hits the first line of sight. Middle: the first line of sight (A) gets disturbed by the passage of the shell. The absorption pattern of A looks very different from that of B. Right: After the shell has slowed down sufficiently pressure waves reestablish equilibrium between the lines of sight. The absorption pattern, although different from the initial state, again looks the same in both lines of sight. 


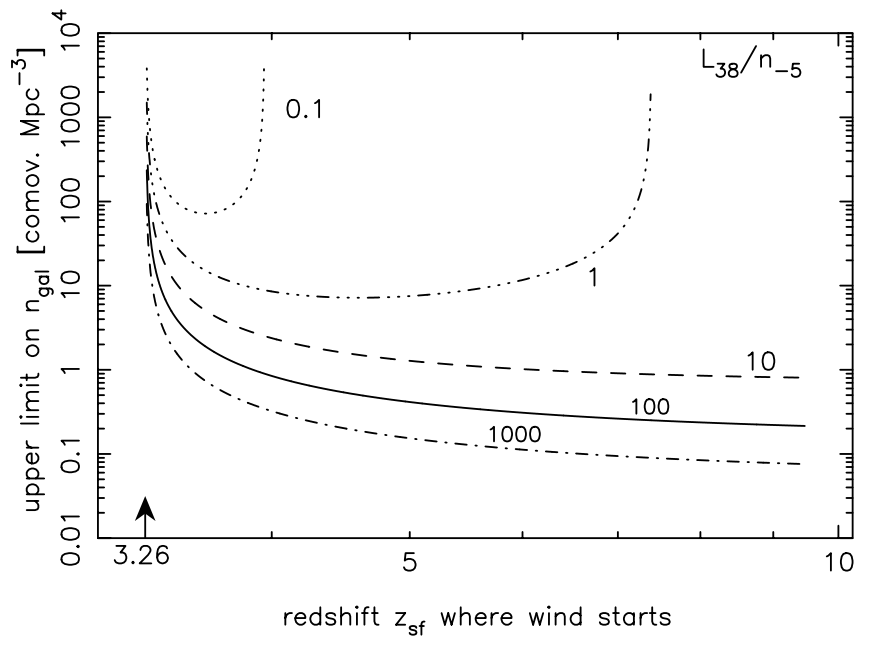

FIG. 11.-Upper limit on the number density of galaxies surrounded by wind bubbles, as a function of the redshift $z_{\mathrm{SF}}$ where the wind starts. The diagram is based on a "disturbed" fraction $f_{\mathrm{LOS}} \leq 0.23$ of the Ly $\alpha$ forest. The different curves belong to different values of $L_{38} / n_{-5}$. Winds at the low end of the $L_{38} / n_{-5}$ range are less well constrained because they have a shorter lifetime for detection. The mean redshift of observation (3.26) is marked by an arrow.

$\mathrm{cm}^{-3}$ ), and the age of the wind bubble $t_{7}$ (in units of $10^{7} \mathrm{yr}$ ). These relations are given in Appendix B.

Figures 11 and 12 show the dependence of the upper limits on $n_{\mathrm{gal}}$ and $f_{v}$ for winds of various "strengths" (i.e., with the ratio $L_{38} / n_{-5}$ as the distinguishing parameter) corresponding to the upper limit $f_{\mathrm{LOS}} \leq 0.23$ on the fraction of disturbed pixels in the Ly $\alpha$ forest.

The model is highly oversimplified in that it assumes spherical bubbles with a single age and a single combination of $L_{38}$ and $n_{\text {gal }}$, propagating through a homogeneous medium (and the expansion of the universe is not taken into account), but it contains several features likely to be relevant to more realistic future models (Figs. 11 and 12):

1. The number of galaxies with winds originating close to the epoch of observation $(z=3.26)$ is essentially uncon-

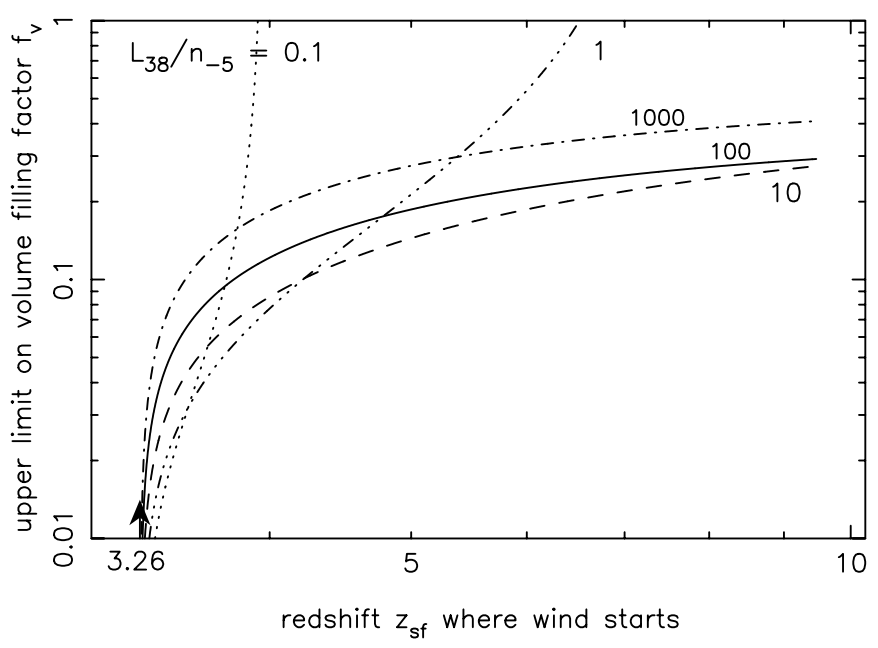

FIG. 12.-Upper limit on the volume filling factor for windblown bubbles. The input parameters are the same as for the previous figure. Strong winds $L_{38} / n_{-5} \sim 100-1000$ can fill a significant fraction of the volume of the universe (up to $40 \%$ if they start as early as redshift 10, up to $\sim 18 \%$ if they start at $z=4$ ), whereas winds with $L_{38} / n_{-5} \leq 1$ are essentially unconstrained if they occur before redshift 7 . strained by an observation of the line-of-sight filling factor (Fig. 11). This is because the shell radii are so small that even a large number of galaxies with winds would produce a negligible total absorption cross section.

2. Weak winds (low luminosity and/or large density of ambient medium, i.e., $L_{38} / n_{-5} \leq 1$ ), because of their limited lifetime, escape detection if they originated early (e.g., before $z_{\mathrm{SF}} \sim 4$ for a $L_{38} / n_{-5} \sim 0.1$ shell, or before $z_{\mathrm{SF}} \sim 7$ for a $L_{38} / n_{-5} \sim 1$ shell; Fig. 11).

3. Strong winds $\left(L_{38} / n_{-5}>10\right)$ remain detectable even if they started a Hubble time ago (Fig. 11), and the upper limit on their numbers becomes stronger with increasing redshift $z_{\mathrm{SF}}$.

4. The volume filling factor (Fig. 12) is most tightly constrained for recent rather than old winds, independent of strength.

5. Our observation cannot exclude that the universe is filled with ancient, weak wind bubbles because they would no longer be detectable with the present method by redshift $z \sim 3$ (see item 2 above). However, since these weak winds do not reach far out from galaxies and galaxies are not distributed randomly, it is very unlikely that such bubbles could fill a substantial fraction of the voids. Strong winds $\left(L_{38} / n_{-5}>10\right)$ are much better constrained because of their long lifetimes: if winds of strength $L_{38} / n_{-5} \sim 1000$ started as early as $z_{\mathrm{SF}} \sim 10$, they could be filling up to about $40 \%$ of the volume of the universe by redshift $z=3.26$. If such strong winds arose at $z_{\mathrm{sf}} \sim 4$, they may fill up to $18 \%$.

\section{CONCLUSIONS}

We have applied various methods to search for and measure density and velocity structure on $100 \mathrm{pc}$ scales in the IGM at $\langle z\rangle=3.26$. The smallness of the differences in column density and velocity between the lines of sight confirm and extend the results of Smette et al. (1992, 1995), and Petry et al. (1998). In the unsaturated regions of the Ly $\alpha$ forest rms differences between the projected line-of-sight velocities, over $120 \mathrm{pc}$ do not exceed a few hundred meters per second. The rms total density differences are inferred to be less that $3 \%$ and are basically below the detection limit. Visible differences between the lines of sight do occur occasionally but are localized and seem to be mostly limited to high column density gas. Strong differences are known to occur from studies of the higher column density metal absorption lines (Paper I and Paper II), but these differences rarely show up in the Ly $\alpha$ region proper because of saturation. Our results appear broadly consistent with predictions by Theuns et al. (2001), who model the impact of stellar feedback on the appearance of the Ly $\alpha$ forest and find that stellar feedback will mostly affect the higher column density, Lyman limit absorption systems. The suggestion (Chen et al. 2000) that most absorption lines with a rest frame equivalent width greater than $0.32 \AA$ (corresponding to $N(\mathrm{H} \mathrm{I}) \sim 6 \times 10^{13} \mathrm{~cm}^{-2}$ for a Doppler parameter $b=28 \mathrm{~km} \mathrm{~s}^{-1}$ ) are related to galactic halos (where we might expect to see hydrodynamic disturbances) is not contradicted by our results because close to $90 \%$ of the $\mathrm{Ly} \alpha$ forest lines above our chosen column density threshold $\left(10^{12} \mathrm{~cm}^{-2}\right)$ have lower column densities.

Our results are consistent with unsaturated Ly $\alpha$ systems being mostly featureless at the few percent level on scales smaller by an order of magnitude than state-of-the-art 
cosmological hydrosimulations can currently resolve. Apparently, the finite resolution of the simulations does not lead to a significant underestimate of the clumpiness of the baryon distribution for densities typical of the unsaturated Ly $\alpha$ forest, i.e., overdensities up to a factor of $10-15$. However, our observations only provide a snapshot of the properties of the Ly $\alpha$ forest at $z \sim 3.3$ and cannot exclude the existence of earlier nongravitational processes in the IGM if, by the time of observation, their hydrodynamic traces have been obliterated (see below).

Notwithstanding doubts about the applicability of the Kolmogorov law to the low-density IGM, we have tentatively derived the rate of turbulent energy input into the low-density IGM. The turbulent energy in the unsaturated low column density $\operatorname{Ly} \alpha$ clouds $\left[\log N\left(\begin{array}{ll}\mathrm{H} & \mathrm{I})<14.13\end{array}\right]\right.$ appears to be lower by 3-4 orders of magnitude than that in the stronger, $\mathrm{C}$ IV absorbing clouds dealt with in Paper II. Apparently, the C IV gas has been affected more recently (and thus more frequently, given it is unlikely that there is a sharply defined "epoch" of star formation) by stellar feedback than the general Ly $\alpha$ forest.

The fraction of the spectra that differ by more than 5\% in optical depth with respect to the other line of sight, considering all saturated regions as being disturbed, was measured for various column density ranges. The results imply a limit on the relative importance of hydrodynamic dis- turbances in the presence of the restoring effects of gas pressure. From the upper limit to the line-of-sight filling factor $\left(f_{\text {Los }}<23 \%\right)$ we find upper limits to the volume filling factor and space density of wind-producing galaxies as a function of the mechanical wind luminosity, the density of the ambient IGM, and the starting redshift of the wind. The presence of short-lived, weak winds (low luminosity or high ambient density) is only poorly constrained. The other extreme, long-lived superwinds with $L_{38} / n_{-5} \sim 1000$ arising as early as redshift 10 , could fill up to $40 \%$ of the volume of the universe by redshift 3.3 , but not more than $18 \%$ if starting at redshift 4 . Currently, there is no observational evidence for the existence of $z \sim 10$ superwinds, but closer to the epoch of our observations strong outflows have been seen in high-redshift galaxies (Franx et al. 1997; Pettini et al. 2001), so there is the possibility that a significant fraction of the volume of the universe may have been stirred by winds; our results appear to show that it is not a dominant one.

M. R. is grateful to NASA for supporting the initial phases of this work under HF-01075.01-94A, and to the NSF for grant AST 00-98492. The work of W. L. W. S. was supported by NSF grant AST 99-00733. We thank Martin Haehnelt, Joop Schaye, and an anonymous referee for helpful suggestions and comments.

\section{APPENDIX A}

Our criterion for the optimum choice of the maximum permissible measurement error (§ 3.2) is as follows: we would like to

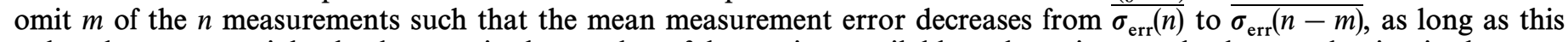
reduced error outweighs the decrease in the number of data points available and continues to lead to a reduction in the error of the determination of the total width of the distribution to be measured, $\sigma_{\mathrm{tot}}(n) /(n)^{1 / 2} \rightarrow \sigma_{\mathrm{tot}}(n-m) /(n-m)^{1 / 2}$; in other words, we throw away the data points with the largest errors until the uncertainty in the width of the distribution does not improve anymore. Data points should be continued to be omitted as long as

$$
E=[n-1+S(m)] \overline{\sigma_{\mathrm{err}}^{2}(n)}-\frac{n(n-1)}{n-m} \overline{\sigma_{\mathrm{err}}^{2}(n-m)}-\sigma_{\mathrm{tot}}^{2}(n) S(m)>0,
$$

where

$$
S(m)=\sum_{k=0}^{m-1} \frac{n(n-1)}{(n-k)(n-k-1)} .
$$

For $m=1$, we obtain the simple relation

$$
\overline{\sigma_{\mathrm{err}}^{2}(n)}-\overline{\sigma_{\mathrm{err}}^{2}(n-1)}>\frac{\sigma_{\mathrm{tot}}^{2}(n)}{n} .
$$

The function $E$ is shown for the distributions of the velocity and column density differences in the bottom panels of Figures 5 and 8. The optimum value of $E$ occurs where $E$ changes from positive to negative values.

\section{APPENDIX B}

This appendix addresses two questions: (1) would a superbubble or wind blowing into the IGM at high redshift produce detectable $\mathrm{H}$ I absorption, and (2) can we produce limits on the volume filling factor of extragalactic wind bubbles from the fraction of the $L y \alpha$ forest spectrum disturbed by such events?

\section{B1. DETECTION OF H I ABSORPTION FROM DENSE SHELLS IN THE IGM}

We assume that the dynamics of the wind shell can be described by the superbubble model of Mac Low \& McCray (1988). Then the dependence of the radius $R$ on the mechanical luminosity $L=L_{38} \times 10^{38} \mathrm{ergs} \mathrm{s}^{-1}$, the time $t=t_{7} \times 10^{7} \mathrm{yr}$, and the 
number density of protons $n=n_{-5} 10^{-5} \mathrm{~cm}^{-3}$ in the ambient IGM, is given by

$$
R=L_{38}^{1 / 5} n_{-5}^{-1 / 5} t_{7}^{3 / 5} 2670 \mathrm{pc},
$$

and the expansion velocity is

$$
\dot{R}=L_{38}^{1 / 5} n_{-5}^{-1 / 5} t_{7}^{-2 / 5} 157 \mathrm{~km} \mathrm{~s}^{-1} .
$$

The total hydrogen column density seen radially outward through the shell is

$$
N_{s}(\mathrm{H})=n_{s} \Delta R=\frac{n R}{3}=2.75 \times 10^{16} L_{38}^{1 / 5} n_{-5}^{4 / 5} t_{7}^{3 / 5} \mathrm{~cm}^{-2} .
$$

The gas density $n_{s}$ in the shell is given by (Weaver et al. 1977):

$$
n_{s}=n \frac{\dot{R}^{2}+c^{2}}{c_{s}^{2}},
$$

where $c$ is the sound speed in the undisturbed IGM and $c_{s}$ is the sound speed in the shell. The thickness of the shell, $\Delta R$, is

$$
\Delta R=\frac{N_{s}}{n_{s}}=\frac{n R}{3 n_{s}}=\frac{R c_{s}^{2}}{3\left(\dot{R}^{2}+c^{2}\right)}=14.4 \frac{L_{38}^{1 / 5} n_{-5}^{-1 / 5} t_{7}^{3 / 5} c_{s, 20}^{2}}{L_{38}^{2 / 5} n_{-5}^{-2 / 5} t_{7}^{-4 / 5}+0.0162 c_{20}^{2}} \mathrm{pc}
$$

The sound speeds are measured in units of $20 \mathrm{~km} \mathrm{~s}^{-1}$. The density in the shell is then

$$
n_{s}=\frac{6.16 \times 10^{-4} L_{38}^{2 / 5} n_{-5}^{3 / 5} t_{7}^{4 / 5}+c_{20}^{2}}{c_{s, 20}^{2}} \mathrm{~cm}^{-3} .
$$

It is not clear how exactly the gas is ionized. If it were in photoionization equilibrium with an ionizing UV background radiation field with intensity $J=10^{-21} \mathrm{ergs} \mathrm{cm}^{-2} \mathrm{~s}^{-1} \mathrm{~Hz}^{-1} \mathrm{sr}^{-1}$, the neutral fraction would be approximately (Bergeron \& Stasinska 1986):

$$
\frac{n(\mathrm{H} \mathrm{I})}{n(\mathrm{H} \mathrm{II})}=1.67 \times 10^{-3}\left(\frac{n_{s}}{10^{-2} \mathrm{~cm}^{-3}}\right) .
$$

Thus, the $\mathrm{H}$ I column density of the shell (from eqs. [B3] and [B7]) is

$$
N_{s}(\mathrm{H} \mathrm{I})=\frac{n(\mathrm{H} \mathrm{I})}{n(\mathrm{H} \mathrm{II})} N_{s}(\mathrm{H})=2.8 \times 10^{12} n_{-5}^{7 / 5} L_{38}^{3 / 5} t_{7}^{7 / 5} c_{s, 20}^{-2}\left[1+0.0162 c_{20}^{2} n_{-5}^{2 / 5} L_{38}^{-2 / 5} t_{7}^{-4 / 5}\right] \mathrm{cm}^{-2},
$$

which is well detectable in absorption in our data. The actual absorption is likely to be stronger (the bubble certainly sweeps up material with higher than average density $\left(n_{-5} \sim 1\right.$ at $\left.z \sim 3\right)$ in the vicinity of the galaxy from which it originates.

\section{B2. EXPANDING SHELLS AS REVEALED BY SMALL-SCALE DIFFERENCES IN THE ABSORPTION PATTERN}

A dense shell crossing two adjacent lines of sight separated by a distance $\Delta r$ will produce differences in the column densities as long as the time it takes the shell to traverse the lines of sight is shorter than the sound crossing time $\Delta r / c\left(=5.8 \times 10^{6} \mathrm{yr}\right.$, in the present case). From simple geometric arguments,

$$
\frac{\dot{R}}{R} b>c,
$$

where $b$ is the impact parameter of the lines of sight relative to the center of the shell. Thus, the shell is detectable for $b>b_{\min }=c R / \dot{R}$, and the cross section $\sigma$ of the shell for producing absorption lines with differences between the lines of sight that have not been ironed out by pressure waves is

$$
\sigma= \begin{cases}\pi R^{2}\left(1-\frac{c^{2}}{\dot{R}^{2}}\right) & \text { for } \dot{R}>c \\ 0 & \text { for } \dot{R} \leq c .\end{cases}
$$

The "footprint" of the shell absorption in the Ly $\alpha$ forest spectrum is expected to consist of a pair of absorption lines, and we assume here that each of these is broadened with a velocity width $\Delta v_{\mathrm{fwhm}}=46 \mathrm{~km} \mathrm{~s}^{-1}$ (Doppler parameter $28 \mathrm{~km} \mathrm{~s}^{-1}$, which is typical for a photoionized Ly $\alpha$ line in the $z=3$ IGM). Conservatively, we count only the sum of the widths of the two lines as contribution to the disturbance in the Ly $\alpha$ forest spectrum, as it is not clear whether the interior of the bubble would produce detectable structure between the lines of sight (it is most likely to produce a clearing in the forest without small-scale features).

When expressed in spatial coordinates (at $z=3.26$ ) the combined velocity widths of the two lines corresponds to a proper distance $\Delta L=2 \Delta v_{\mathrm{fwhm}} H(z)^{-1} \approx 206 h_{50}^{-1} \mathrm{kpc}$. The fraction of the Ly $\alpha$ forest spectrum disturbed by a coeval population of galaxies with space density $n_{\text {gal }}$, producing identical, spherically symmetric, expanding wind shells with radii $R$, and expansion 
velocities $\dot{R}$ would be

$$
f_{\mathrm{LOS}}=n_{\mathrm{gal}} \sigma \Delta L=2 n_{\mathrm{gal}} \pi R^{2}\left(1-\frac{c^{2}}{\dot{R}^{2}}\right) \frac{\Delta v_{\mathrm{fwhm}}}{H}, \quad \text { for } \dot{R}>c,
$$

whereas the volume filling factor for the wind bubbles is simply

$$
f_{v}=n_{\mathrm{gal}} \frac{4 \pi}{3} R^{3}=\frac{2}{3} f_{\mathrm{LOS}} R\left(1-\frac{c^{2}}{\dot{R}^{2}}\right)^{-1} \frac{H}{\Delta v_{\mathrm{fwhm}}} .
$$

Using the Mac Low \& McCray superbubble model to get a first crude estimate, the quantities $n_{\text {gal }}$ and $f_{v}$ can be expressed as functions of the line-of-sight covering factor $f_{\text {Los }}$, the time $t_{7}$ or redshift $z_{\text {sf }}$ when the wind (or star-forming episode) started, and the ratio between the mechanical luminosity and the gas density in the IGM, $L_{38} / n_{-5}$. The upper limits on $n_{\text {gal }}$ and $f_{v}$ corresponding to the upper limit $f_{\mathrm{LOS}} \leq 0.23$ are shown as a function of $z_{\mathrm{sf}}$ for various values of the parameter $L_{38} / n_{-5}$ in Figures 11 and 12, respectively. The transformation between time and redshift assumes $\Omega_{m}=0.3$ and $H_{0}=50 \mathrm{~km} \mathrm{~s}^{-1} \mathrm{Mpc}^{-1}$.

\section{REFERENCES}

Aguirre, A., Hernquist, L., Schaye, J., Weinberg, D. H., Katz, N., \& Gardner, J. 2001, ApJ, in press

Barlow, T. A., \& Sargent, W. L. W. 1997, AJ, 113, 136

Bechtold, J., \& Yee, H. K. C. 1995, AJ, 110, 1984

Bergeron, J., \& Stasinska, G. 1986, A\&A, 169, 1

Bryan, G. L., Machacek, M., Anninos, P., \& Norman, M. L. 1999, ApJ, 517, 13

Carswell, R. F., Lanzetta, K. M., Parnell, H. C., \& Webb, J. K. 1991, ApJ, 371,36

Cen, R., \& Bryan, G. L. 2001, ApJ, 546, L81

Cen, R., Miralda-Escudé, J., Ostriker, J. P., \& Rauch, M. 1994, ApJ, 437, L9

Chen, H.-W., Lanzetta, K. M., \& Fernández-Soto, A. 2000, ApJ, 533, 120

Couchman, H. M. P., \& Rees, M. J. 1986, MNRAS, 221, 53

Croft, R. A., Weinberg, D. H., Katz, N., \& Hernquist, L. 1997, ApJ, 488, 532

Davé, R., Hernquist, L., Katz, N., \& Weinberg, D. H. 1999, ApJ, 511, 521

Ferrara, A., Pettini, M., \& Shchekinov, Y. 2000, MNRAS, 319, 539

Foltz, C. B., Weymann, R. J., Röser, H. J., \& Chaffee, F. H. 1984, ApJ, 281, 1

Franx, M., Illingworth, G. D., Kelson, D. D., van Dokkum, P. G., \& Tran, K.-V. 1997, ApJ, 486, L75

Hernquist, L., Katz, N., Weinberg, D., \& Miralda-Escudé, J. 1996, ApJ, 457, L51

Kaplan, S. A., \& Pilkener, S. B. 1970, The Interstellar Medium (Cambridge: Harvard Univ. Press)

Kundic, T., Hogg, D. W., Blandford, R. D., Cohen, J. G., Lubin, L. M., \& Larkin, J. E. 1997, AJ, 114, 2276

Lopez, S., Reimers, D., Rauch, M., Sargent, W. L. W., \& Smette, A. 1999, ApJ, 513, 598

Mac Low, M.-M., \& McCray, R. 1988, ApJ, 324, 776

Madau, P., Ferrara, A., \& Rees, M. J. 2001, ApJ, 555, 92

Miralda-Escudé, J., Cen, R., Ostriker, J. P., \& Rauch, M. 1996, ApJ, 471, 582

Patnaik, A. R., Browne, I. W. A., Walsh, D., Chaffee, F. H., \& Foltz, C. B. 1992, MNRAS, 259, 1
Peebles, P. J. E. 1993, Principles of Physical Cosmology (Princeton: Princeton Univ. Press)

Petitjean, P., Muecket, J. P. \& Kates, R. E. 1995, A\&A, 295, L9

Petry, C. E., Impey, C. D., \& Foltz, C. B. 1998, ApJ, 494, 60

Pettini, M., Shapley, A., Steidel, C. C., Cuby, J.-G., Dickinson, M., Moorwood, A. P. E., Adelberger, K. L., \& Giavalisco, M. 2001, ApJ, 554, 981

Rauch, M., Carswell, R. F., Chaffee, F. H., Foltz, C. B., Webb, J. K., Weymann, R. J., Bechtold, J., \& Green, R. F. 1992, ApJ, 390, 387

Rauch, M., et al. 1997, ApJ, 489, 7

Rauch, M., Sargent, W. L. W., \& Barlow, T. A. 1999, ApJ, 515, 500 (Paper I)

. 2001, ApJ, 554, 823 (Paper II)

Sargent, W. L.W., Young, P. J., Boksenberg, A., \& Tytler, D. 1980, ApJS, 42,41

Schaye, J. 2001, ApJ, 559, 507

Smette, A., Robertson, J. G., Shaver, P. A., Reimers, D., Wisotzki, L., \& Koehler, T. 1995, A\&AS, 113, 199

Smette, A., Surdej, J., Shaver, P. A., Foltz, C. B., Chaffee, F. H., Weymann, R. J., Williams, R. E., \& Magain, P. 1992, ApJ, 389, 39

Theuns, T., Leonard, A., Efstathiou, G., Pearce, F. R., \& Thomas, P. A. 1998, MNRAS, 301, 478

Theuns, T., Mo, H. J., \& Schaye, J. 2001, MNRAS, 321, 450

Vogt, S. S., et al. 1994, Proc. SPIE, 2198, 362

Wadsley, J., \& Bond, J. R. 1996, in ASP Conf. Ser. 123, Computational Astrophysics, ed. D. A. Clarke \& M. J. West (San Francisco: ASP), 332

Weaver, T. A., McCray, R., Castor, J., Shapiro, P., \& Moore, R. 1977, ApJ, 218,377

Webb, J. K. 1987, Ph.D. thesis, Cambridge Univ.

Weinberg, D. H. Miralda-Escudé, J., Hernquist, L., \& Katz, N. 1997, ApJ, 490,564

Weymann, R. J., \& Foltz, C. B. 1983, ApJ, 272, L1

Young, P. J., Sargent, W. L. W., Boksenberg, A., \& Oke, J. B. 1981, ApJ, 249,415

Zhang, Y., Anninos, P., \& Norman, M. L. 1995, ApJ, 453, L57

Zhang, Y., Anninos, P., Norman, M. L., \& Meiksin, A. 1997, ApJ, 485, 496 